\title{
Crystal structure and magnetic properties of $\mathrm{GdSi}_{1.78}$, $\mathbf{G d}\left(\mathrm{Si}_{0.684} \mathbf{G e}_{0.316}\right)_{1.78}, \mathbf{G d G e}_{1.57}$, and $\mathrm{GdSn}_{2}$ compounds
}

\author{
J. D. Zou ${ }^{\mathrm{a}, \mathrm{b}, *}, \mathrm{~J} . \mathrm{Liu}^{\mathrm{b}, \mathrm{c}}$, and M. Yan ${ }^{\mathrm{a}, *}$ \\ a School of Materials Science and Engineering, State Key Laboratory of Silicon \\ Materials, Key Laboratory of Novel Materials for Information Technology of Zhejiang \\ Province, Zhejiang University, Hangzhou, Zhejiang 310027, China \\ ${ }^{\mathrm{b}}$ The Ames Laboratory, US Department of Energy, Iowa State University, Ames, Iowa \\ 50011-3020, USA \\ ${ }^{c}$ Department of Materials Science and Engineering, Iowa State University, Ames, Iowa \\ 50011-2300, USA
}

* Corresponding author. Tel.:+86 571 87952366, fax:+86 57187952366

E-mail address: zoujd@zju.edu.cn (J. D. Zou),mse_yanmi@zju.edu.cn (M. Yan) 


\begin{abstract}
:
Intermetallic compounds of $\mathrm{Gd}$ with $\mathrm{Si}, \mathrm{Ge}$, and $\mathrm{Sn}$ near 1:2 stoichiometry adopt several closely related crystal structures. We find that $\mathrm{GdSi}_{1.78}$ and $\operatorname{Gd}\left(\mathrm{Si}_{0.684} \mathrm{Ge}_{0.316}\right)_{1.78}$ crystallize in the same $\mathrm{GdSi}_{1.4}$-type orthorhombic structure (space group Imma), while $\mathrm{GdGe}_{1.57}$ and $\mathrm{GdSn}_{2}$ adopt $\alpha$-ThSi ${ }_{2}$-type tetragonal structure (space group $\mathrm{I}_{1} / \mathrm{amd}$ ) and $\mathrm{ZrSi}_{2}$-type orthorhombic structure (space group $\mathrm{Cmcm}$ ), respectively. All compounds order antiferromagnetically; their Néel temperatures are only weakly affected by the magnetic field of less than $50 \mathrm{kOe}$. Unusual features are observed including multiple phase transitions and thermomagnetic irreversibilities.
\end{abstract}

Keywords: rare earth intermetallics; magnetic properties; phase transition; antiferromagnetism; heat capacity. 


\section{INTRODUCTION}

Intermetallic compounds formed by lanthanide metals with the elements of group 14 in the periodic system have been attracting continuous attention since 1960's when relatively pure lanthanides have become commercially available, and especially so after the discovery of giant magnetocaloric,,${ }^{1,2}$ magnetoresistance, ${ }^{3,4}$ and magnetostriction ${ }^{5}$ effects in Gd-rich $\mathrm{Gd}_{5} \mathrm{Si}_{4-\mathrm{x}} \mathrm{Ge}_{\mathrm{x}}$ compounds. The latter adopt three different yet closely related structures at room temperature depending on composition (x), namely the $\mathrm{Gd}_{5} \mathrm{Si}_{4^{-}}$ type, $\mathrm{Gd}_{5} \mathrm{Si}_{2} \mathrm{Ge}_{2}$-type, and $\mathrm{Sm}_{5} \mathrm{Ge}_{4}$-type structures. ${ }^{6,7,8}$ The magnetic and structural phase transitions that occur in the $\mathrm{Gd}_{5} \mathrm{Si}_{4-\mathrm{x}} \mathrm{Ge}_{\mathrm{x}}$ compounds can be controlled by changing chemical composition, magnetic field, temperature, and/or applied pressure. 1,2, 9, 10 Experimental and theoretical studies show that most of the interesting phenomena observed in $\mathrm{Gd}_{5} \mathrm{Si}_{4-\mathrm{x}} \mathrm{Ge}_{\mathrm{x}}$ compounds are associated with field (temperature or pressure)induced first-order structural transitions between paramagnetic (PM) monoclinic or antiferromagnetic (AFM) orthorhombic and ferromagnetic (FM) orthorhombic structures. ${ }^{11}$

Gd-poor compounds, $\mathrm{GdT}_{2}(\mathrm{~T}=\mathrm{Si}, \mathrm{Ge}$, and $\mathrm{Sn})$, in some aspects, are similar to the 5:4type compounds, especially with respect to their polymorphism. It has been reported that the 1:2 compounds adopt several closely related crystal structures, including $\mathrm{GdSi}_{1.4}$-type and $\mathrm{ZrSi}_{2}$-type orthorhombic (space groups Imma or Cmcm), ${ }^{12,13,14,15,16,17} \alpha$-ThSi ${ }_{2}$-type tetragonal (space group $I 4_{1} / a m d$ ), ${ }^{18,19,20}$ and $\mathrm{AlB}_{2}$-type hexagonal structures (space group $P 6 / \mathrm{mmm}){ }^{21}$ Figure 1 shows the unit cells of three structures which will be discussed in this work. The bonds between Gd and the elements in group 14 are omitted. Earlier studies show that $\mathrm{GdSi}_{2}$ has very low Schottky-barrier height suggesting potential 
applications in infrared detectors. ${ }^{22}$ Nanowires, a highly anisotropic form of nanocrystals, have attracted considerable interest due to their unusual physical properties and potential applications in nanoscale devices and interconnections. ${ }^{23}$ Growth of $\mathrm{GdSi}_{2}$ nanowires on a silicon substrate, $\mathrm{Si}(100)$, has been reported. ${ }^{24,25,26,27}$ Yet even today, these Gd-based compounds are not well understood, and their fundamental properties should be further studied.

The 1:2-type rare earth compounds $\mathrm{RT}_{2}(\mathrm{R}=$ rare earth, $\mathrm{T}=$ group 14 element $)$ show rich magnetism. At low temperature, light lanthanide compounds (such as $\mathrm{CeGe}_{2}, \mathrm{PrGe}_{2}$, $\mathrm{NdGe}_{2}$, and $\mathrm{PrSi}_{2}$ ) are ferromagnets, while heavy lanthanide compounds are antiferromagnets (the Néel temperatures, $T_{N}$, do not follow the de Gennes law) or Pauli paramagnets. ${ }^{12,16,19}$ In the past, basic magnetic properties of orthorhombic $\mathrm{GdSi}_{2}$ and $\mathrm{GdGe}_{2}$ were studied: they order AFM at 27 and $28 \mathrm{~K}$, respectively. ${ }^{12,16}$ The effective magnetic moments of $\mathrm{Gd}$ in $\mathrm{GdSi}_{2}$ and $\mathrm{GdGe}_{2}$ are 7.81, and $8.1 \mu_{\mathrm{B}} / \mathrm{Gd}^{3+}$, respectively, which are close to $7.94 \mu_{\mathrm{B}}$ - theoretical value of the free ion. The high temperature $\mathrm{x}$-ray diffraction (XRD) confirms that $\mathrm{GdSi}_{2}$ undergoes a transformation from tetragonal to orthorhombic structure at $\sim 818 \mathrm{~K} .{ }^{14}$ First principles electronic structure calculations (performed without accounting for the interatomic $4 f$ interactions and $f-d$ hybridization) reveal that the $\mathrm{AFM}$ state of $\mathrm{GdSi}_{2}$ is energetically more stable than the FM state in any of the three possible structures. ${ }^{28}$ No detailed magnetic properties of $\mathrm{GdSn}_{2}$ have been reported.

\section{EXPERIMENT DETAILS}

The Gd metal was prepared by the Materials Preparation Center of the Ames Laboratory and was 99.93 wt.\% pure with respect to all other elements in the periodic 
table. $^{29}$ The major impurities were O (0.018 wt.\%), Yb (0.002 wt.\%), Al (0.015 wt.\%), C (0.017 wt.\%), and N (0.012 wt.\%). The purities of Si, Ge, and $\mathrm{Sn}$ were better than 99.99 wt.\%. $\mathrm{GdSi}_{2}, \mathrm{GdSiGe}, \mathrm{GdGe}_{2}$ and $\mathrm{GdSn}_{2}$ samples with the stoichiometric 1:2 (R:T) compositions were prepared by arc melting pure elements under argon gas atmosphere on a water-cooled copper hearth. The $\mathrm{GdGe}_{2}$ and $\mathrm{GdSn}_{2}$ specimens were separately sealed in quartz tubes backfilled with pure helium gas, and then annealed at $1423 \mathrm{~K}$ for 21 days and $1073 \mathrm{~K}$ for 14 days, respectively. These two samples were quenched in ice-water mixture after annealing procedure. Crystal structures of phases presenting in the samples were determined from room-temperature x-ray powder diffraction (XRD) data collected using a PANalytical X'Pert Pro powder diffractometer employing monochromatic $\mathrm{Cu}$ $K \alpha_{1}$ radiation (wavelength of $1.54056 \AA$ ). The lattice parameters, phase concentrations, and other structural parameters were determined by performing Rietveld refinement using Rietica-LHPM. ${ }^{30}$

Magnetization measurements were conducted by using the magnetic properties measurement system MPMS XL-7, Quantum Design, Inc. The measurements were performed in the temperature range of $5-40 \mathrm{~K}$ in applied dc magnetic fields up to $50 \mathrm{kOe}$. A homemade adiabatic heat-pulse calorimeter was used to conduct the heat-capacity measurements. ${ }^{31}$ The measurements were performed in the temperature range from $\sim 2$ to $50 \mathrm{~K}$ in zero magnetic field and in applied magnetic fields up to $50 \mathrm{kOe}$.

\section{RESULTS AND DISCUSSIONS}

\section{A. Crystal structure}

The observed and calculated room-temperature XRD patterns are displayed in Fig.2, and the structural parameters are summarized in Table 1. According to earlier reports, 
$\mathrm{GdSi}_{2}$ can crystallize in orthorhombic, ${ }^{12,13}$ tetragonal, ${ }^{18,19}$ and hexagonal structures. ${ }^{21}$ The as-prepared $\mathrm{GdSi}_{2}$ and $\mathrm{GdSiGe}$ crystallize in the orthorhombic crystal structure (space group Imma), which is an orthorhombically distorted variant of the tetragonal $\alpha-\mathrm{ThSi}_{2^{-}}$ type structure. The Rietveld refinement results (Fig. 2(a), 2(b) and Table 1) show that these two samples contain about $7 \mathrm{wt} \%$ of $\mathrm{Si}$ and $\mathrm{Si}-\mathrm{Ge}$ phases, respectively. Both samples are nonstoichiometric compounds, and the actual ratio between $\mathrm{Gd}$ and $\mathrm{Si}$ (or $\mathrm{Si}$ Ge) is 1:1.78, as determined from Rietveld refinement of site occupancies. The orthorhombic structure remains stable after 21 days heat treatment at $1423 \mathrm{~K}$, but the concentrations of the impurity phases (pure $\mathrm{Si}$ or $\mathrm{Si}-\mathrm{Ge}$ ) somewhat increase.

For the $\mathrm{GdGe}_{2}$ compound, previous studies indicate that it adopts the orthorhombic structure after annealing under vacuum. ${ }^{15,16}$ Our sample adopts tetragonal $\alpha$-ThSi 2 -type structure (space group $I 4_{1} /$ amd) after annealing at $1423 \mathrm{~K}$ for 21 days, see Fig. 1(b) and 2(c). This sample also contains atomic defects with an accurate composition of $\mathrm{GdGe}_{1.57}$. Annealing at lower temperature, e.g. $973 \mathrm{~K}$, is insufficient to achieve the single phase. In addition to $\mathrm{Ge}$ (far less than 1 wt.\%), our $\mathrm{GdGe}_{1.57}$ sample contains about 6 wt.\% of $\mathrm{Gd}_{3} \mathrm{Ge}_{5}$ which adopts the face-centered $\mathrm{Y}_{3} \mathrm{Ge}_{5}$-type orthorhombic structure with space group Fdd2. ${ }^{32,33}$ The as-prepared $\mathrm{GdSn}_{2}$ sample crystallizes in the orthorhombic structure (space group $\mathrm{Cmcm}$ ). The results of Rietveld refinement of the heat-treated $\mathrm{GdSn}_{2}$ are shown in Fig 2(d) and Table 1. This heat-treated $\mathrm{GdSn}_{2}$ sample contains about 3 wt.\% of Sn metal as a minor impurity. Compared with the as-cast sample, the heat treatment at $1073 \mathrm{~K}$ for 14 days is beneficial in reducing the concentration of Sn impurity. Unlike the nonstoichiometric $\mathrm{GdSi}_{1.78}$ and $\mathrm{GdGe}_{1.57}$ compounds, we note that $\mathrm{GdSn}_{2}$ is a stoichiometric compound. 


\section{B. Magnetic properties}

Although the phase volume monotonically increases in the series $\operatorname{GdSi}_{1.78}$, $\operatorname{Gd}\left(\mathrm{Si}_{0.684} \mathrm{Ge}_{0.316}\right)_{1.78}, \mathrm{GdGe}_{1.57}$, and $\mathrm{GdSn}_{2}$, as expected, considering the difference in the atomic volumes of $\mathrm{Si}, \mathrm{Ge}$, and $\mathrm{Sn}$, the magnetic properties exhibit a more complicated behavior. The magnetic measurements show that all four samples order antiferromagnetically. The temperature dependence of magnetization of $\mathrm{GdSi}_{1.78}$, shown in Fig. 3, indicates AFM ordering below $25 \mathrm{~K}$. The Néel temperature $\left(T_{N}\right)$ of $\mathrm{GdSi}_{1.78}$ is close to previous reports. ${ }^{12,}{ }^{34}$ Besides the main AFM transition, two anomalies are observed below $T_{N}$; they are marked as $T^{\prime}$ and $T^{\prime \prime}$. Thermomagnetic irreversibility between zero-field-cooling (ZFC) and field-cooling (FC) measurements is observed between $14 \mathrm{~K}$ and $T=20 \mathrm{~K}$ in $1 \mathrm{kOe}$. The thermal hysteresis region shifts toward higher temperature at $50 \mathrm{kOe}$. In addition, the ZFC and FC curves in $50 \mathrm{kOe}$ show a substantial thermal hysteresis down to the lowest measurement temperature and the ZFC curve shows an anomaly at $T=8 \mathrm{~K}$. The $\mathrm{Gd}\left(\mathrm{Si}_{0.684} \mathrm{Ge}_{0.316}\right)_{1.78}$ sample crystallizes in the same structure as $\mathrm{GdSi}_{1.78}$, but its $T_{N}$ decreases to $22 \mathrm{~K}$ as a result of Ge substitution (Fig. 4). Weak thermal hysteresis is observed below $10 \mathrm{~K}$ in low fields, and the thermal hysteresis becomes more prominent with the increasing magnetic field. Similar to $\mathrm{GdSi}_{1.78}$, an anomaly is also observed at $T "=7 \mathrm{~K}$.

Although the annealed $\mathrm{GdGe}_{1.57}$ crystallizes in the tetragonal structure, which is different from $\mathrm{GdSi}_{1.78}$ and $\mathrm{Gd}\left(\mathrm{Si}_{0.684} \mathrm{Ge}_{0.316}\right)_{1.78}, T_{N}$ of $\mathrm{GdGe}_{1.57}$ further decreases to $18 \mathrm{~K}$ which is lower than the reported value of the orthorhombic $\operatorname{GdGe}_{2}\left(T_{N}=28 \mathrm{~K}\right),{ }^{16}$ see Fig.

5. The minor break of the slope at $25 \mathrm{~K}$ is possibly due to the impurity phase of $\mathrm{Gd}_{3} \mathrm{Ge}_{5} .{ }^{34}$ The thermal hysteresis behavior of the germanide is different from that of $\mathrm{GdSi}_{1.78}$ and 
$\mathrm{Gd}\left(\mathrm{Si}_{0.684} \mathrm{Ge}_{0.316}\right)_{1.78}$. The thermal hysteresis of $\mathrm{GdGe}_{1.57}$ compound begins at $T_{N}$ in $1 \mathrm{kOe}$. With the increase of the magnetic field, the start point of thermal hysteresis drop below $15 \mathrm{~K}$, and the hysteresis regions keep decreasing.

Compared with $\mathrm{GdSi}_{1.78}$ and $\mathrm{Gd}\left(\mathrm{Si}_{0.684} \mathrm{Ge}_{0.316}\right)_{1.78}, \mathrm{GdSn}_{2}$ compound also adopts an orthorhombic structure, but it belongs to a different space group (Cmcm). $T_{N}$ of $\mathrm{GdSn}_{2}$ increases to $28 \mathrm{~K}$ (Fig. 6) which is higher than $T_{N}$ of $\mathrm{GdSi}_{1.78}$. Thermal hysteresis can be observed at the lowest temperatures, and it persists up to $50 \mathrm{kOe}$. Thermal hysteresis features in all four samples most likely suggest the existence of magnetic clustering which originate from the layered structure. Note that the extended $5 \mathrm{~d}$ bands of $\mathrm{Si}, \mathrm{Ge}$, and Sn might offer a small FM component which also can lead to thermomagnetic irreversibility behaviors in all title samples. ${ }^{35}$ In addition to the AFM ordering transition at $T_{N}$, another anomaly is observed at $T^{\prime}=21 \mathrm{~K} . T^{\prime}$ of $\mathrm{GdSn}_{2}$ does not obviously change with the applied magnetic field which is different from that in $\mathrm{GdSi}_{1.78}$.

The effective magnetic moment $p_{\text {eff }}$ of $\mathrm{Gd}$ in $\mathrm{GdSi}_{1.78}$ and $\mathrm{GdSn}_{2}$ are $8.19 \mu_{\mathrm{B}}$ and $8.16 \mu_{\mathrm{B}}$, respectively, which are larger than the theoretical value of $7.94 \mu_{\mathrm{B}}$ for free trivalent ion $\mathrm{Gd}^{3+}$, but are actually very close to the usually observed values. $p_{\text {eff }}$ of $\mathrm{GdGe}_{1.57}$ is $7.98 \mu_{\mathrm{B}}$ which is almost the same as the theoretical value. $p_{\text {eff }}$ of $\operatorname{Gd}\left(\mathrm{Si}_{0.684} \mathrm{Ge}_{0.316}\right)_{1.78}$ is $7.69 \mu_{\mathrm{B}}$ which is lower than the expected value. It is well known that the $4 f-4 f$ exchange interactions in rare-earth elements are mediated by conduction electrons $(s p d)$. The $4 f-4 f$ exchange interactions spin split the conduction electrons (mainly the $5 d$ electrons). The $\mathrm{Si}, \mathrm{Ge}$ and $\mathrm{Sn}$ in $\mathrm{GdSi}_{1.78}, \mathrm{Gd}\left(\mathrm{Si}_{0.684} \mathrm{Ge}_{0.316}\right)_{1.78}$ and $\mathrm{GdSn}_{2}$ would be hybridized in both spin directions by Gd atoms leading to strong $5 d-p$ hybridization. Therefore, the deviation of magnetic moment to the theoretical value can 
be quite possibly ascribed to strong $5 d-p$ hybridization. In addition, the deviation also possibly originate from the spin density wave (SDW) state and magnetic polaron of extended $5 \mathrm{~d}$ bands of $\mathrm{Si}, \mathrm{Ge}$, and $\mathrm{Sn}$ induced by localized $4 \mathrm{f}$ moments. ${ }^{35,36}$ The Néel temperature $T_{N}$ decreases from $25 \mathrm{~K}\left(\mathrm{GdSi}_{1.78}\right)$ to $22 \mathrm{~K}\left(\mathrm{Gd}\left(\mathrm{Si}_{0.684} \mathrm{Ge}_{0.316}\right)_{1.78}\right)$, to $18 \mathrm{~K}$ $\left(\mathrm{GdGe}_{1.57}\right)$, then increases to $28 \mathrm{~K}\left(\mathrm{GdSn}_{2}\right)$. The paramagnetic Weiss temperature $\theta_{p}$ increases from $-73 \mathrm{~K}\left(\mathrm{GdSi}_{1.78}\right)$ to $-54 \mathrm{~K}\left(\mathrm{Gd}\left(\mathrm{Si}_{0.684} \mathrm{Ge}_{0.316}\right)_{1.78}\right)$, to $-39 \mathrm{~K}\left(\mathrm{GdGe}_{1.57}\right)$, and then decreases to $-69 \mathrm{~K}\left(\mathrm{GdSn}_{2}\right)$. The changing tendency of $T_{\mathrm{N}}$ and the absolute value of $\theta_{p}$ match with each other very well. Usually, it is expected that $T_{N}$ and $\left|\theta_{p}\right|$ should decrease with the expansion of unit cell volume, such as the cases of $\mathrm{GdSi}_{1.78}$, $\mathrm{Gd}\left(\mathrm{Si}_{0.684} \mathrm{Ge}_{0.316}\right)_{1.78}$, and $\mathrm{GdGe}_{1.57}$. However, $T_{N}$ and $\left|\theta_{p}\right|$ of $\mathrm{GdSn}_{2}$ anomaly increase.

\section{Heat-capacities}

We have performed heat-capacity measurements in order to further explore the observed magnetic phase transitions. Figure 7 show the heat-capacities, $C_{p}$, of all four samples measured as a function of temperature in zero and $50 \mathrm{kOe}$ magnetic fields. The heat-capacity of $\mathrm{GdSi}_{1.78}$ only shows one peak at $T_{N}=25 \mathrm{~K}$, see Fig. 7(a). The heatcapacity behavior of $\mathrm{Gd}\left(\mathrm{Si}_{0.684} \mathrm{Ge}_{0.316}\right)_{1.78}$ is similar with that of $\mathrm{GdSi}_{1.78}$ except that the peak shifts downward to $22 \mathrm{~K}$, shown in Fig. 7(b). Figure 7(c) shows the heat-capacity of $\mathrm{GdGe}_{1.57}$, and a small peak is observed at $T_{N}=18 \mathrm{~K}$. Another anomaly appears at $25 \mathrm{~K}$ due to the orthorhombic impurity phase. As for $\mathrm{GdSn}_{2}$, two phase transitions are confirmed at $21 \mathrm{~K}$ and $28 \mathrm{~K}$, respectively, as shown in Fig. 7(d). The positions of the two peaks correspond with $T^{\prime}$ and $T_{N}$ in the temperature dependence magnetization, as shown in Fig. 6. Compared with the $C_{p}$ curves in zero field, the heat-capacity peaks of all samples slightly yet clearly move to low temperatures and the height of these peaks goes down a 
little in $50 \mathrm{kOe}$ which might be signs of magnetic field induced phase transitions. It should be noted that anomalous increases are observed in $C_{p}$ curves below around $15 \mathrm{~K}$ for all samples. This behavior might originate from the anomalous increase in the magnetic part of specific heat as a function of temperature while it is independent on the magnetic field less than $50 \mathrm{kOe}$.

\section{CONCLUSIONS}

Although $\mathrm{GdSi}_{1.78}, \mathrm{Gd}\left(\mathrm{Si}_{0.684} \mathrm{Ge}_{0.316}\right)_{1.78}, \mathrm{GdGe}_{1.57}$ and $\mathrm{GdSn}_{2}$ compounds adopt different structures, all compounds are AFM ordering. Moreover, the unit cell volumes monotonically increase with the increase in atomic number, while $T_{N}$ decreases from 25 $\mathrm{K}$ for $\mathrm{GdSi}_{1.78}$ to $18 \mathrm{~K}$ for $\mathrm{GdGe}_{1.57}$, then increase to $27 \mathrm{~K}$ for $\mathrm{GdSn}_{2}$. In addition to AFM transitions, two anomalies are observed in $\mathrm{GdSi}_{1.78}$ in magnetic measurements, while these anomalies cannot be observed in $C_{p}$ curves. $\mathrm{GdSn}_{2}$ shows another magnetic phase transition below $T_{N}$ confirmed by both magnetic and heat-capacity measurements. All compounds show the thermal hysteresis between ZFC and FC curves below $T_{N}$, but the thermal hysteresis in $\mathrm{GdSi}_{1.78}$ and $\mathrm{GdGe}_{1.57}$ are prominent in particular.

\section{ACKNOWLEDGEMENT}

This work was supported by the National Natural Science Foundation of China (Grant No. 51471150), and Program for Innovative Research Team in University of Ministry of Education of China (IRT13R54). Work at the Ames Laboratory was supported by the U. S. Department of Energy, Office of Basic Energy Science, Division of Materials Sciences and Engineering. The research was performed at the Ames Laboratory operated for the U. 
S. Department of Energy by Iowa State University under Contract No. DE-AC02$07 \mathrm{CH} 11358$.

1 V. K. Pecharsky and K. A. Gschneidner, Jr., Phys. Rev. Lett. 78, 4494 (1997).

${ }^{2}$ V. K. Pecharsky and K. A. Gschneidner, Jr., Appl. Phys. Lett. 70, 3299 (1997).

${ }^{3}$ L. Morellon, J. Stankiewicz, B. Garcia-Landa, P. A. Algarabel, and M. R. Ibarra, Appl. Phys. Lett. 73, 3462 (1998).

${ }^{4}$ E. M. Levin, V. K. Pecharsky, and K. A. Gschneidner, Jr., Phys. Rev. B 60, 7993 (1999).

${ }^{5}$ L. Morellon, P. A. Algarabel, M. R. Ibarra, J. Blasco, B. Garcia-Landa, Z. Arnold, and F. Albertini, Phys. Rev. B 58, R14721 (1998).

${ }^{6}$ K. A. Gschneidner, Jr., V. K. Pecharsky, and A. O. Tsokol, Rep. Prog. Phys. 68, 1479 (2005).

7 V. K. Pecharsky and K. A. Gschneidner, Jr., J. Alloys Compd. 260, 98 (1997).

8 V. K. Pecharsky and K. A. Gschneidner, Jr., Adv. Mater. 13, 683 (2001).

${ }^{9}$ C. Magen, L. Morellon, P. A Algarabel, M. R. Ibarra, Z. Arnold, J. Kamarad, T. A. Lograsso, D. L. Schlagel, V. K. Pecharsky, A. O. Tsokol, and K. A. Gschneidner, Jr., Phys. Rev. B 72, 024416 (2005).

10 L. Morellon, Z. Arnold, P. A. Algarabel, C. Magen, M. R. Ibarra, and Y. Skorokhod, J. Phys.: Condens. Matter 16, 1623 (2004).

11 Y. Mudryk, V.K. Pecharsky, and K.A. Gschneidner, Jr., in Handbook on the Physics and Chemistry of Rare Earths, edited by J.-C. Bünzli and V.K. Pecharsky (Elsevier B.V., Amsterdam, 2014), Vol. 44, p.283. 
12 K. Sekizawa and K. Yasukochi, J. Phys. Soc. Jpn. 21, 274 (1966).

13 J. Pierre, E. Siaud, and D. Frachon, J. Less-Common Met. 139, 321 (1988).

${ }^{14}$ F. X. Zhang, P. Limermann, H. X. Yang, and S. K. Saxena, J. Phys.: Condens. Matter 16, 7787 (2004).

15 E. I. Gladyshevskii and V. V. Burnashova, Inorg. Mater. (Engl. Transl.) 1, 1374 (1965).

16 K. Sekizawa, J. Phys. Soc. Jpn. 21, 1137 (1966).

17 A. Iandelli, A. Palenzona, and G. B. Bonino, Atti Accad. Naz. Lincei, Cl. Sci. Fis., Mat. Nat., Rend., (in Italian) 40, 623 (1966).

18 J. A. Perri, E. Banks, and B. Post, J. Phys. Chem. 63, 2073 (1959).

19 K. Yaguchi, J. Phys. Soc. Jpn. 21, 405 (1966).

20 I. Mayer and Y. Eshdat, Inorg. Chem. 7, 1904 (1968).

21 A. Iandelli, A. Palenzona, and G. L. Olcese, J. Less-Common Met. 64, 213 (1979).

${ }^{22}$ K. N. Tu, R. D. Thompson, and B. Y. Tsaur, Appl. Phys. Lett. 38, 626 (1981).

${ }^{23}$ R. F. Service, Science 294, 2442 (2001).

24 Y. Chen, D. A. A. Ohlberg, and R. S. Williams, J. Appl. Phys. 91, 3212 (2002).

25 B. Z. Liu and J. Nogami, Nanotechnology 14, 837 (2003).

${ }^{26}$ H. W. Yeom, Y. K. Kim, E. Y. Lee, K.-D. Ryang, and P. G. Kang, Phys. Rev. Lett. 95, $205504(2005)$.

27 B. C. Harrison and J. J. Boland, Surf. Sci. 594, 93 (2005).

28 W. S. Yun, G. Cha, and S. C. Hong, J. Magn. Magn. Mater. 304, e31 (2006).

${ }^{29}$ Materials Preparation Center, Ames Laboratory US-DOE, Ames, IA, USA, [www.mpc.ameslab.gov]. 
${ }^{30}$ B. Hunter, Rietica-A Visual Rietveld Program, International Unionof Crystallography Commission on Powder Diffraction Newsletter, No. 20 (Summer, 1998), [http://www.rietica.org.]

31 V. K. Pecharsky, J. O. Moorman, and K. A. Gschneidner, Jr., Rev. Sci. Instrum. 68, 4196 (1997).

32 G. Venturini, I. Ijjaali, and B. Malaman, J. Alloys Compd. 285, 194 (1999).

33 P. H. Tobash, D. Lins, S. Bobev, N. Hur, J. D. Thompson, and J. L. Sarrao, Inorg. Chem. 45, 7286 (2006).

${ }^{34}$ J. Pierre, S. Auffret, R. Madar, E. Houssay, A. Rouluat, and J. P. Senateur, J. Magn. Magn. Mater. 89, 86 (1990).

${ }^{35}$ H. F. Li, Y. G. Xiao, B. Schmitz, J. Persson, W. Schmidt, P. Meuffels, G. Roth, and T. Brückel, Sci. Rep. 2, 750; DOI:10.1038/srep00750 (2012).

${ }^{36}$ N. E. Sluchanko, A. L. Khoroshilov, M. A. Anisimov, A. N. Azarevich , A. V. Bogach, V. V. Glushkov, S. V. Demishev, V. N. Krasnorussky, V. V. Voronov, N. Yu. Shitsevalova, V. B. Filippov, A. V. Levchenko, G. Pristas, S. Gabani, K. Flachbart, arXiv:1412.0497 (2014). 
Figure captions:

Figure 1. (Color online) Unit cells of three structures adopted by the title compounds. (a) The $\mathrm{GdSi}_{1.78}$ and $\mathrm{Gd}\left(\mathrm{Si}_{0.684} \mathrm{Ge}_{0.316}\right)_{1.78}$ compounds adopt orthorhombic structure (space group Imma); (b) The GdGe 1.57 adopts the tetragonal structure (space group I4 $/$ amd), (c) The GdSn 2 adopts the orthorhombic structure (space group Cmcm). The coordinates of (a) and (b) are different. The large black spheres represent Gd atoms, and the small red and blue spheres represent $\mathrm{Si}$, Ge, or Sn atoms. For convenience, only the covalent-like T-T bonds are shown.

Figure 2. (Color online) Observed and calculated powder x-ray diffraction patterns for (a) $\mathrm{GdSi}_{1.78}$, (b) $\mathrm{Gd}\left(\mathrm{Si}_{0.684} \mathrm{Ge}_{0.316}\right)_{1.78}$, (c) $\mathrm{GdGe}_{1.57}$ and (d) $\mathrm{GdSn}_{2}$ at room temperature. Data were analysed by Rietveld refinement technique using the program of Rietica-LHPM. The wavelength of $\mathrm{Cu} K \alpha_{1}$ radiation used in the refinement is $1.54056 \AA$.

Figure 3. Temperature dependence of magnetization of $\mathbf{G d S i}_{1.78}$ under different applied magnetic fields. Gray lines and black lines show the results of ZFC and FC, respectively. Dash lines indicate the positions of $T^{\prime}$ and $T_{N}$, respectively.

Figure 4. Temperature dependence of magnetization of $\operatorname{Gd}\left(\mathrm{Si}_{0.684} \mathrm{Ge}_{0.316}\right)_{1.78}$ under different applied magnetic fields. Gray lines and black lines show the results of ZFC and FC, respectively. Dash line indicates the position of $\boldsymbol{T}_{N}$. 
Figure 5. Temperature dependence of magnetization of $\mathrm{GdGe}_{1.57}$ under different applied magnetic fields. Gray lines and black lines show the results of ZFC and FC, respectively. Dash line indicates the position of $\boldsymbol{T}_{N}$.

Figure 6. Temperature dependence of magnetization of $\mathrm{GdSn}_{2}$ under different applied magnetic fields. Gray lines and black lines show the results of ZFC and FC, respectively. Dash lines indicate the positions of $T^{\prime}$ and $T_{N}$, respectively.

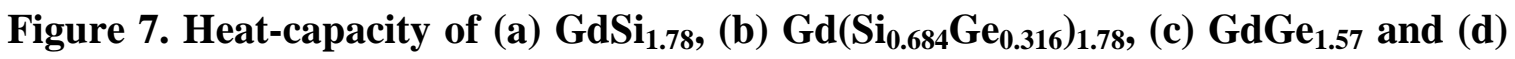
$\mathrm{GdSn}_{2}$ as a function of temperature and magnetic field. The gray lines show the results in zero magnetic field, and the black lines show the results in $50 \mathrm{kOe}$.

Table 1. Chemical formula, space group, lattice parameters, atomic positions and occupations of $\mathrm{GdSi}_{1.78}, \mathrm{Gd}\left(\mathrm{Si}_{0.684} \mathrm{Ge}_{0.316}\right)_{1.78}, \mathrm{GdGe}_{1.57}$ and $\mathrm{GdSn}_{2}$. 


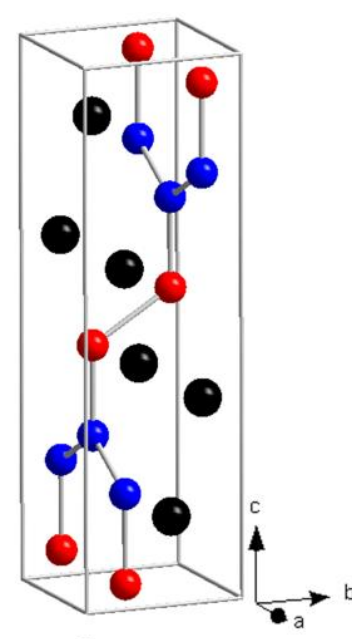

(a) Imma

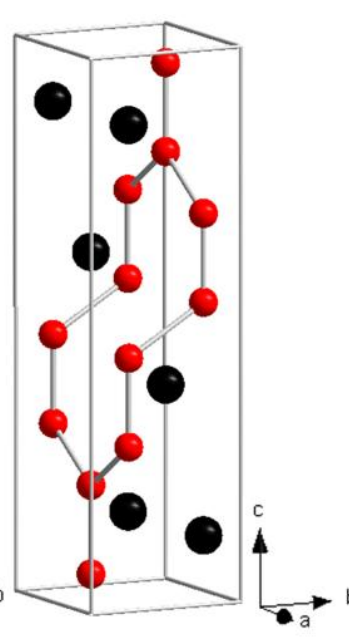

(b) $\mathrm{I} 4_{1} /$ amd

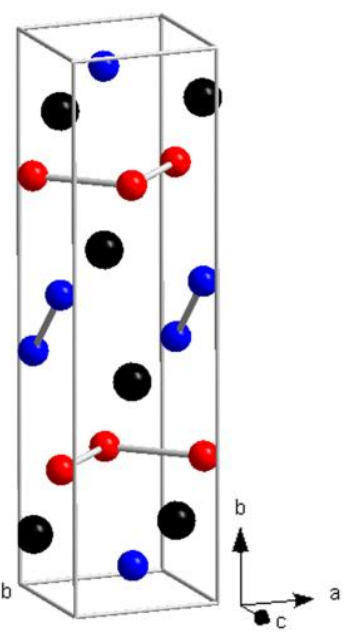

(c) $\mathrm{Cmcm}$

FIG. 1. 

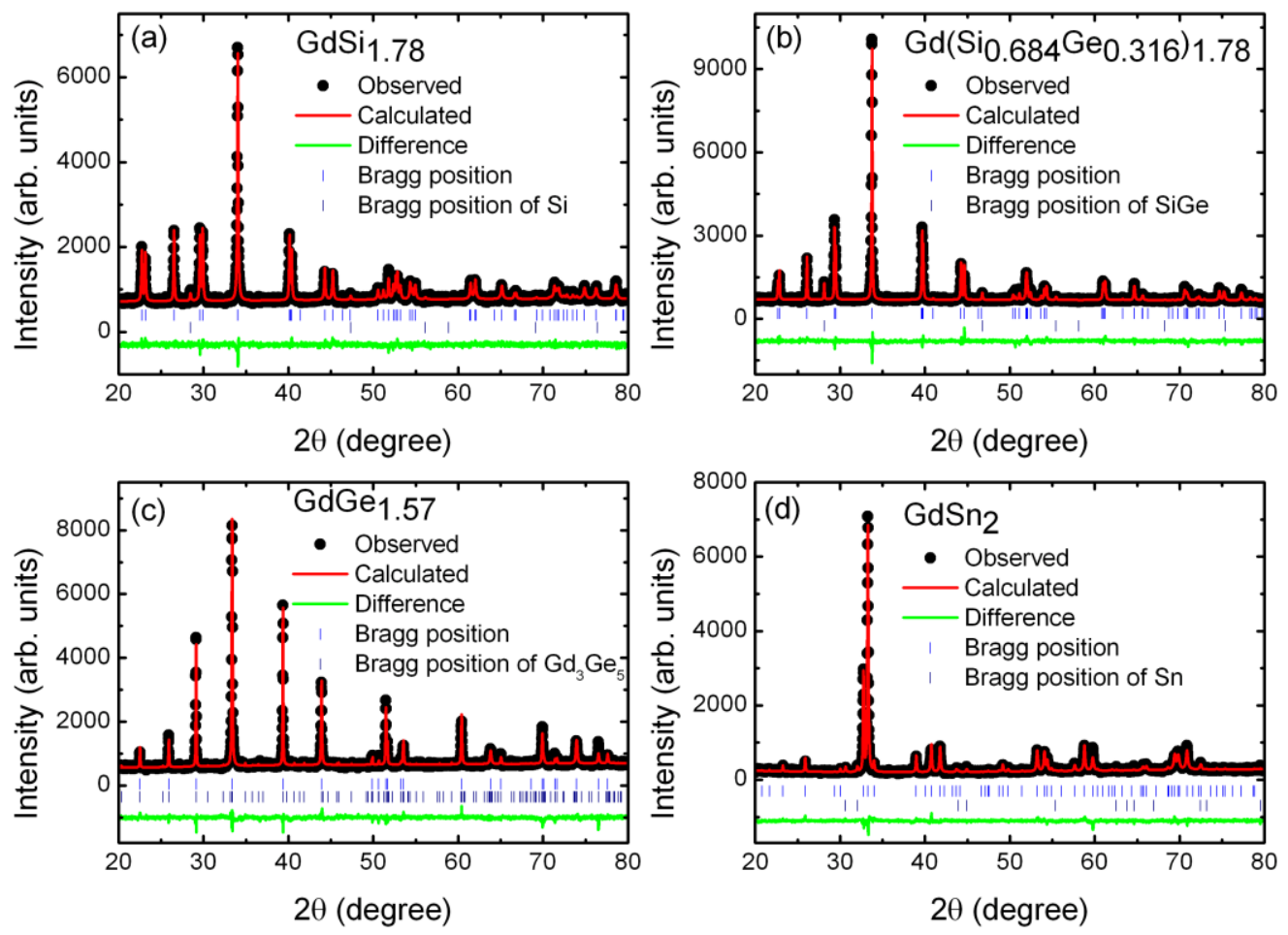

FIG.2 


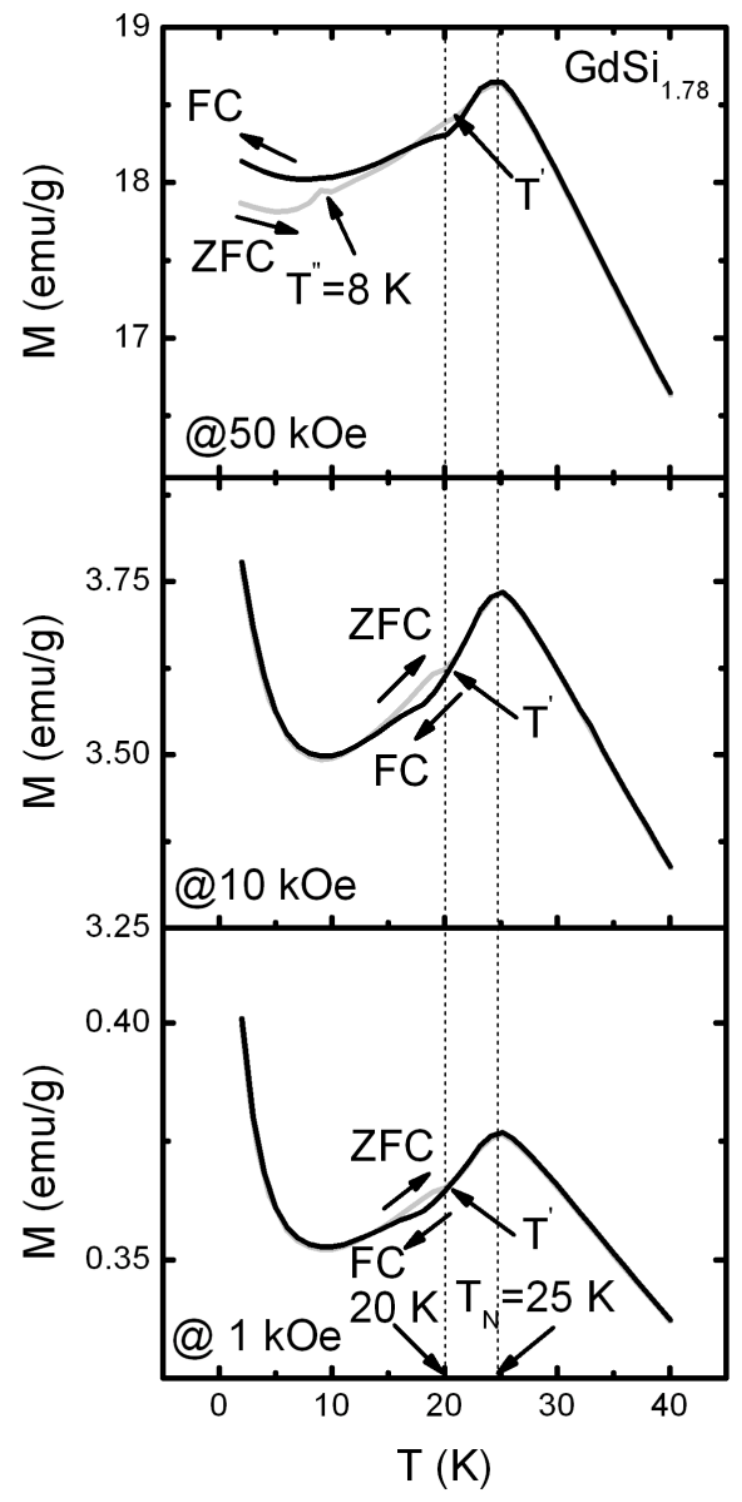

FIG. 3 


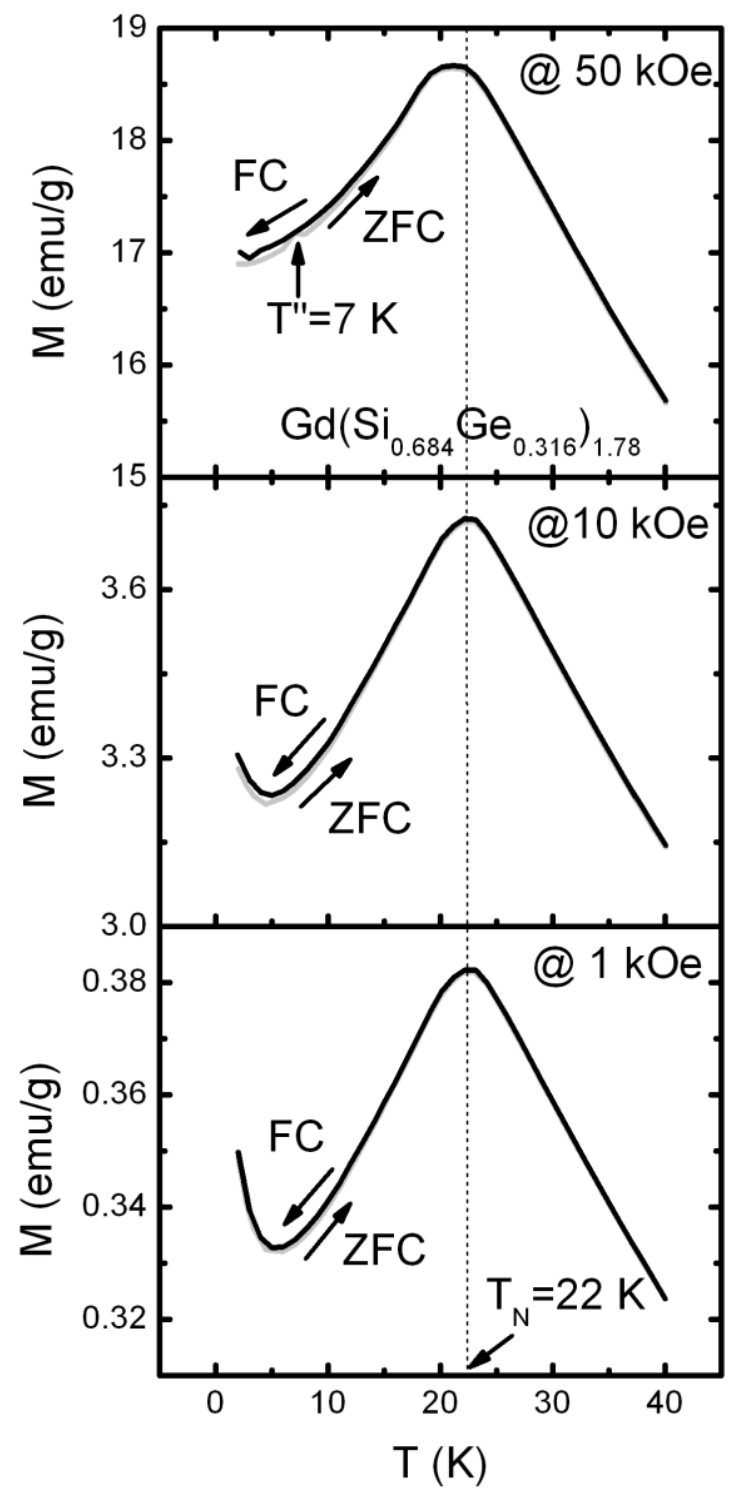

FIG. 4 


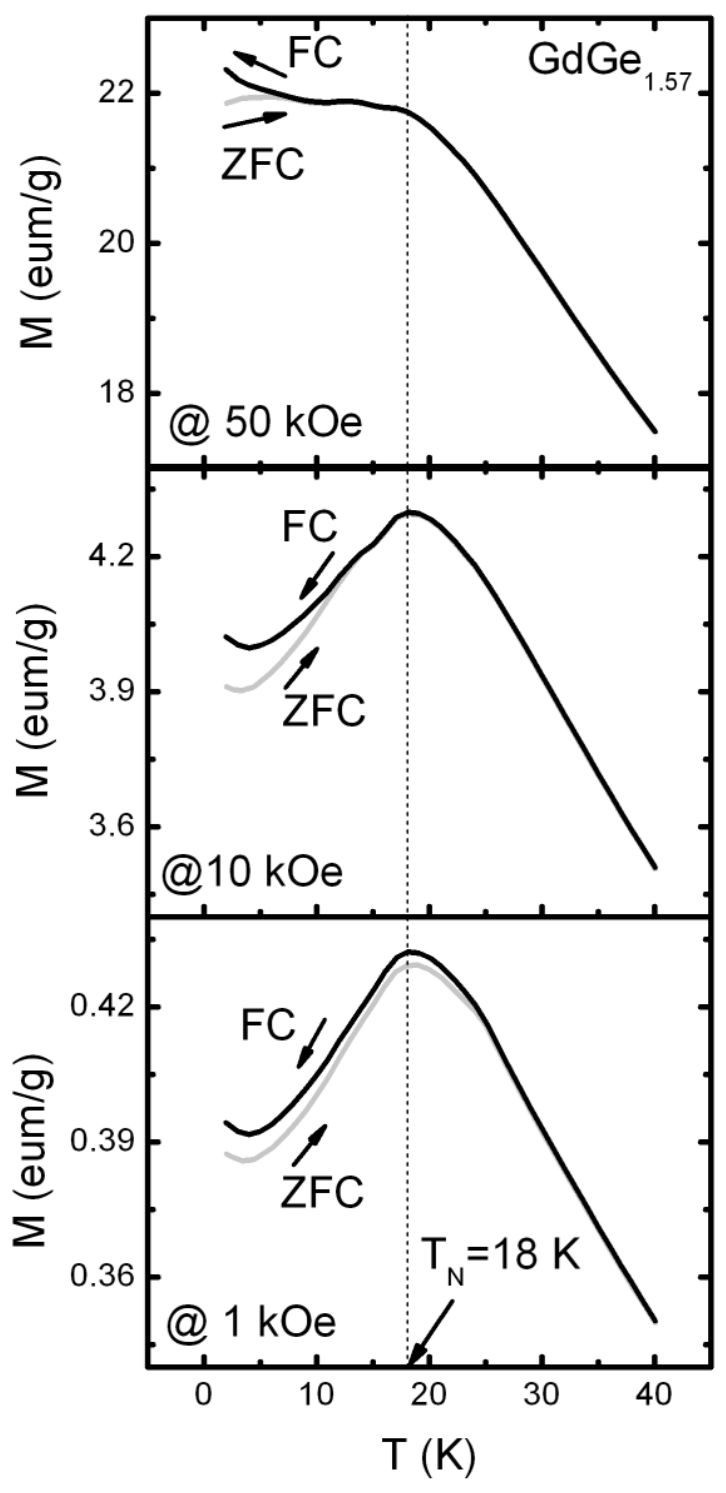

FIG.5 


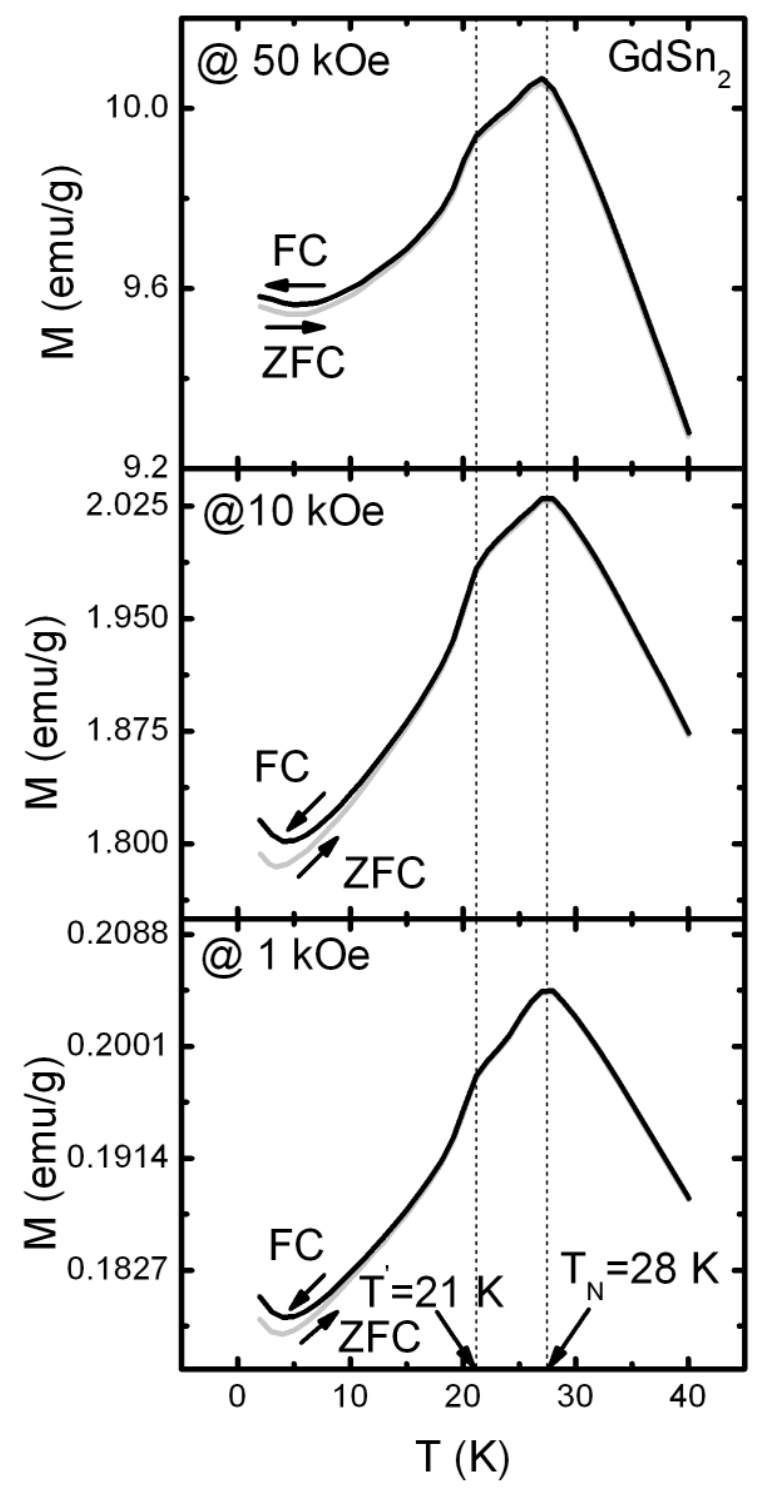

FIG. 6 

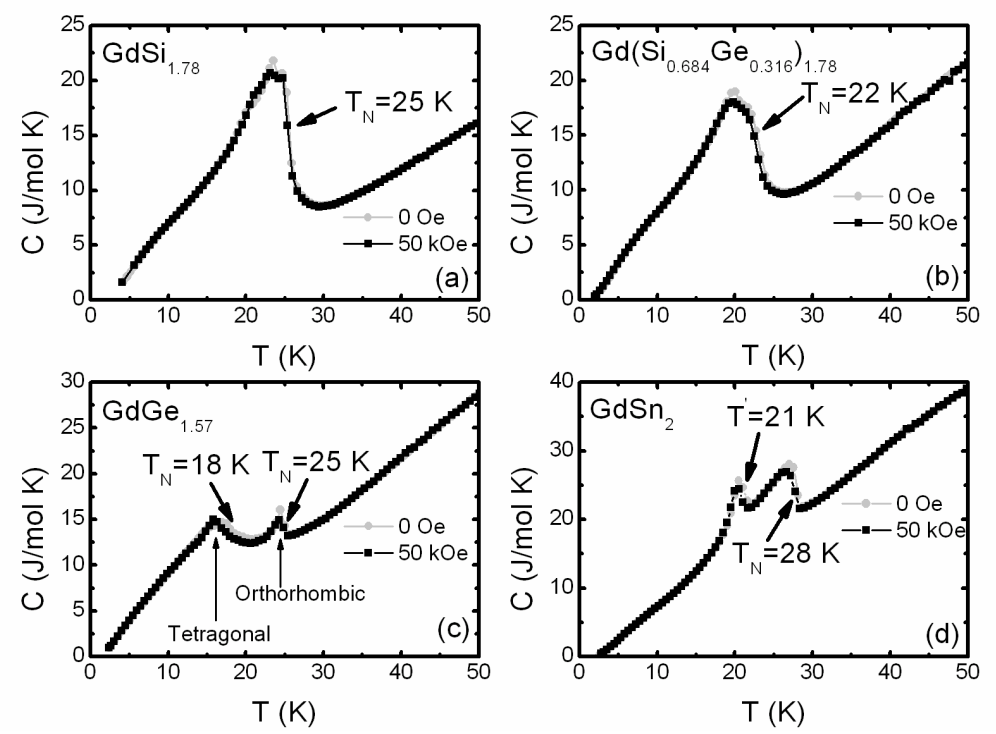

FIG. 7 


\section{Table 1.}

\begin{tabular}{|c|c|c|c|c|c|c|c|}
\hline & Atom & Position & Symmetry & $\mathrm{x} / \mathrm{a}$ & $\mathrm{y} / \mathrm{b}$ & $\mathrm{z} / \mathrm{c}$ & Occupancy \\
\hline \multirow[t]{3}{*}{$\mathrm{GdSi}_{1.78}$} & $\mathrm{Gd}$ & $4 \mathrm{e}$ & $\mathrm{mm} 2$ & 0 & 0.25 & 0.625 & 1.0 \\
\hline & Sil & $4 \mathrm{e}$ & $\mathrm{mm} 2$ & 0 & 0.25 & $0.0488(6)$ & $0.968(3)$ \\
\hline & $\mathrm{Si} 2$ & $4 \mathrm{e}$ & $\mathrm{mm} 2$ & 0 & 0.25 & $0.2142(7)$ & 0.812 \\
\hline S.G. Imma & $\mathrm{a}=4.0090(1) \AA$ & $\mathrm{b}=4.0893(2) \AA$ & $\mathrm{c}=13.4417(4) \AA$ & $\mathrm{v}=220.36(1) \AA^{3}$ & $\mathrm{R}_{\mathrm{p}}=3.14$ & $\mathrm{R}_{\mathrm{wp}}=3.96$ & $\chi^{2}=1.33$ \\
\hline \multirow[t]{5}{*}{$\overline{\mathrm{Gd}}\left(\mathrm{Si}_{0.684} \mathrm{Ge}_{0.316}\right)_{1.78}$} & Gd & $4 \mathrm{e}$ & $\mathrm{mm} 2$ & $\overline{0}$ & 0.25 & $0.6224(3)$ & 1.0 \\
\hline & Sil & $4 \mathrm{e}$ & $\mathrm{mm} 2$ & 0 & 0.25 & $0.0515(5)$ & $0.64(3)$ \\
\hline & $\mathrm{Si} 2$ & $4 \mathrm{e}$ & $\mathrm{mm} 2$ & 0 & 0.25 & $0.2185(5)$ & $0.577(3)$ \\
\hline & Ge1 & $4 \mathrm{e}$ & $\mathrm{mm} 2$ & 0 & 0.25 & $0.0515(5)$ & $0.25(3)$ \\
\hline & $\mathrm{Ge} 2$ & $4 \mathrm{e}$ & $\mathrm{mm} 2$ & 0 & 0.25 & $0.2185(5)$ & $0.313(3)$ \\
\hline S.G. Imma & $\mathrm{a}=4.0583(1) \AA$ & $\mathrm{b}=4.0922(1) \AA$ & $\mathrm{c}=13.6539(4) \AA$ & $\mathrm{v}=226.75(1) \AA^{3}$ & $\mathrm{R}_{\mathrm{p}}=3.60$ & $\mathrm{R}_{\mathrm{wp}}=4.67$ & $\chi^{2}=1.70$ \\
\hline \multirow[t]{2}{*}{$\mathrm{GdGe}_{1.57}$} & $\overline{\mathrm{Gd}}$ & 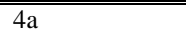 & $-4 m^{2}$ & 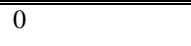 & 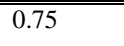 & 0.125 & 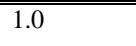 \\
\hline & $\mathrm{Ge}$ & $8 \mathrm{e}$ & $2 \mathrm{~mm}$ & 0 & 0.25 & $0.2936(2)$ & $0.788(2)$ \\
\hline S.G. I4/amd & $\mathrm{a}=4.1234(8) \AA$ & $\mathrm{b}=4.1234(8) \AA$ & $\mathrm{c}=13.7594(3) \AA$ & $\mathrm{v}=233.94(8) \AA^{3}$ & $\mathrm{R}_{\mathrm{p}}=4.11$ & $\mathrm{R}_{\mathrm{wp}}=5.33$ & $\chi^{2}=2.06$ \\
\hline \multirow[t]{3}{*}{$\mathrm{GdSn}_{2}$} & Gd & $4 \mathrm{c}$ & $m 2 \mathrm{~m}$ & 0 & $0.0978(2)$ & 0.25 & 1.0 \\
\hline & Sn1 & $4 \mathrm{c}$ & $\mathrm{m} 2 \mathrm{~m}$ & 0 & 0.75 & 0.25 & 1.0 \\
\hline & $\operatorname{Sn} 2$ & $4 \mathrm{c}$ & $\mathrm{m} 2 \mathrm{~m}$ & 0 & $0.4365(2)$ & 0.25 & 1.0 \\
\hline S.G. $\mathrm{Cmcm}$ & $\mathrm{a}=4.4280(2) \AA$ & $\mathrm{b}=16.4036(6) \AA$ & $\mathrm{c}=4.3230(2) \AA$ & $\mathrm{v}=314.00(2) \AA^{3}$ & $\mathrm{R}_{\mathrm{p}}=5.67$ & $\mathrm{R}_{\mathrm{wp}}=7.35$ & $\chi^{2}=1.66$ \\
\hline
\end{tabular}

